$13^{\text {th }}$ International Conference on

\title{
Analysis and Control of Hybrid Stepper Motor for Automatic Sun-Tracking System
}

\begin{abstract}
A. Sarhan*, Amged El-Wakeel*, A. B. Kotb ${ }^{* *}$
Abstract: With the cost decreasing of photovoltaic (PV) system, the conversion of solar energy to electric energy is increasing becoming economical. In order to increase the efficiency of PV system, it is generally used dynamic sun-tracking system. The goal of the dynamic sun-tracking system is to make PV plates face the sun directly for capturing the maximum irradiation and promoting the system efficiency. This paper presents a dynamic tracker that has an active control strategy of a two-axis sun-tracking system. This control strategy is able to follow the sun with high accuracy without the necessity of either a precise procedure of installation or recalibration. The paper focuses on the analysis and control of hybrid stepper motors to operate in full-step mode with average torque higher than that obtained in classical full-step mode. This has been achieved using the idea of a half-step firing technique. This overcomes the instability associated with half-step mode. The control strategy is executed using a microcontroller and the simulation results are given to verify the validity and reliability of the proposed active sun-tracking system.
\end{abstract}

\section{Introduction}

Renewable energy sources (RES) are considered as a technological option for significantly contributing to the sustainable energy supply in the world. PV energy generates electricity from solar radiation and, at present, represents one of the RES emerging technologies due to the continuous cost reduction and technological progress [1].

To harvest the vast solar energy, it would be desirable if the energy conversion units are simple, reliable, and of low cost and high efficiency [2]. However, the use of the solar PV arrays in simple, reliable, and of low cost and high efficiency will bring a new type of problem: the necessity of high accuracy solar pointing [3].

There are three main types of sun trackers: passive, microprocessor and electro-optically controlled units $[3,4]$.

Passive systems track the sun without any electronic controls or motors. These trackers contain a fluid, such as Freon, within a frame of pipes. When the array is misaligned the sun heats the Freon on one side of the frame more than the other.

This temperature difference causes the heated Freon to evaporate. It may push a piston or may simply flow to the other side of the array and move it by gravity. These trackers are simple but can only provide a moderately accurate tracking.

\footnotetext{
* Egyptian Armed Forces

** Professor, Elect. Engineering Department, Faculty of engineering, Al-Azhar University, Cairo, Egypt
} 
Microprocessor controlled sun-tracking units use mathematical formulae to predict the sun location and need not sense the sunlight. To determine position, they use stepper motors or optical encoders. They are often used in large systems in which one controller controls many arrays. Many microprocessor controlled trackers use electro-optic sensors for self-calibration. They can also be self-calibrated without sensors by using a current maximizing search routine. This type of tracker is highly accurate but requires a very precise installation, often difficult to achieve.

The electro-optical tracker uses the information of some kind of sensor to estimate the real position of sun. It gives very good results during good weather conditions. The operation of this sun tracker has serious problems at cloudy days.

This paper presents a dynamic sun-tracking system that have an active control strategy of a two- axis sun-tracking system. It does not need precise installation or recalibration. The main characters of the proposed system are:

- The DC motors are replaced by stepper motors to get rid of brush wearing and commutation problems.

- Stepper motors are controlled to provide their highest torque without affecting the stability.

- Two-axis sun tracker is executed instead of the conventional one-axis tracker.

- Sun sensor replaced by simpler and cheaper one.

- The system can follow the sun without limit of rotation.

- Using new control strategy built in one mode of operation instead of two modes and this one mode executed in microcontroller.

\section{System Description}

The proposed control strategy consists of PV modules and positioning system. The proposed positioning system is composed of three main subsystems, light dependant resistor (LDR) as a sun-sensor, two stepper motors with their driving circuits as a system driver, and a microcontroller.

\section{Sun Sensor}

The local position of the sun in traditional solar trackers has to be determined from a set of equations and accurate data of location [5]. This leads to a high mechanical cost to avoid, for example, mechanical tolerances and deformations. These solutions imply a high cost of maintenance. The proposed solution is a small low cost module that will increase the system performance. It can be incorporated with an easy installation in the photovoltaic system or in the heliostat and it requires low maintenance. This small low cost module is a sun sensor. The sun-sensor is designed to determine the position of the sun in sensor elevation and azimuth coordinates [6]. 

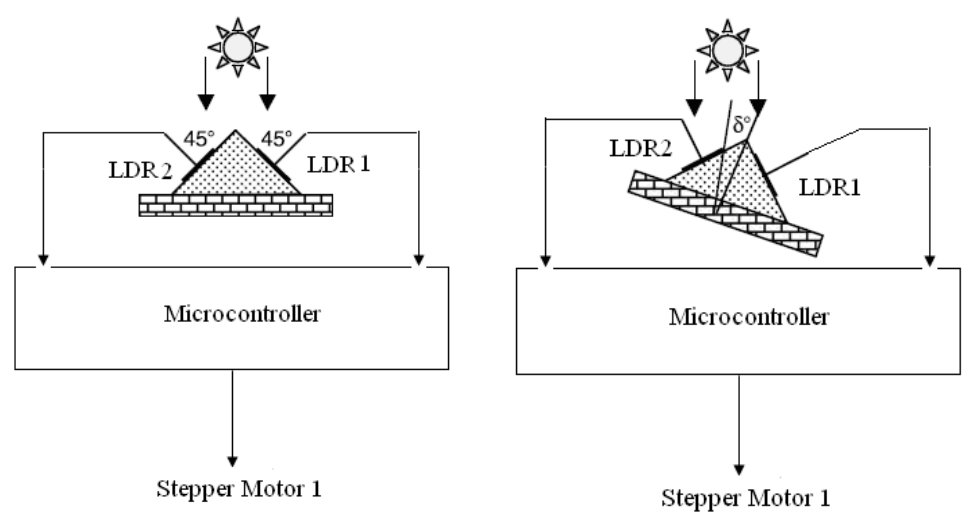

Figure 1 Sun pointing actuator principles.

The principle involved in the design of the automatic tracking system is to sense the sun light with two LDRs. The differential signal representing the angular error of the panel is used to rotate the panel in such a way that the angular error is minimized.

Figure 1 shows the schematic diagram of the differential light sensor mechanism which is used to detect the direction of the sun. Four LDRs are used as the light sensor unit and they are fixed on a four sided pyramid structure and connected to the microcontroller. As shown in Figure 2, when both LDRs are equally illuminated, their respective resistances are the same and hence the voltage applied across them is equal. When any of the LDRs falls in shadow, the voltage applied across them is not the same and hence the microcontroller activates the motor in such direction to bring both LDRs under even illumination.

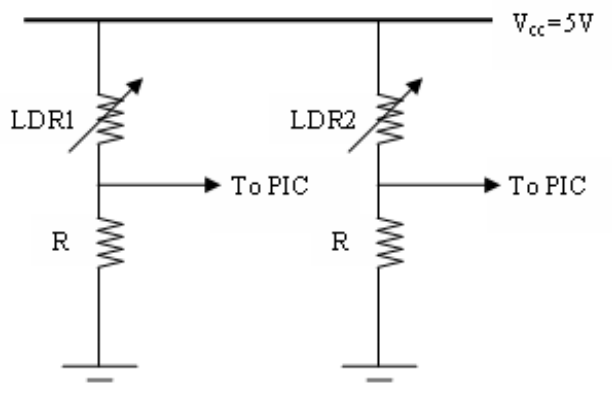

Figure 2 Circuit diagram of the LDR

\section{System Driver}

Orientation drive units in dynamic sun tracker are responsible for positioning the solar array normal to the sun for extracting maximum power. These mechanisms consist of a one-axis or two-axis gimbals, and a motor/controller drive system. The drive motors are configured to provide sufficient torque to autonomously track the sun during the arrays life as well as to reach a faster slew rate when desired. The gimbals mechanism must provide the required strength and stiffness to accommodate all array loads. Drive motors for these devices have evolved throughout the years.

The positioning systems have been traditionally implemented using DC motors due to the relative ease in controlling them [7]. This ease of control is due to the fact that the system equations describing a (separately excited) DC motor are linear. However, there are still disadvantages in using such motors for positing systems. In particular, for high speed repetitive motion, the brushes are subject to excessive mechanical wear and consequently lead 
to a decrease in performance. Also, due to the repetitive motion there is a problem of armature winding overheating. Since the winding are on the rotor, the heat does not have a direct path to the outside environment, but instead must be dissipated through the stator. Finally, the torque to inertia ratio is relatively low, again due to having the armature winding on the rotor. For the reasons just enumerated, the proposed positioning system is implemented using hybrid stepper motors. Hybrid stepper motors are the ideal choice for those applications where power is small and position control is required to be sharp and fast. Due to the lack of rotor windings, the rotor weight, and inertia are highly reduced.. Moreover, reliability, lack of contact aging, mechanical ruggedness, and availability of torque at zero speed are attractive features for aerospace and servo applications.

In general, stepper motor operates in full-step, half-step, or micro-step mode depending on the application. The full-step and half-step modes are generally used for most applications of stepper motors. Half-step mode of operation is normally not envisaged in step motors due to the fact that the torque available for acceleration in succeeding steps will be different and hence will cause different disturbance levels [8]. In the proposed system, the stepper motor will operate in the full-step mode but with firing technique depends on exciting two phases at each step. This result in increasing the torque compared to that obtained in normal full-step and overcomes the instability problem associated with half-step technique.

The electromechanical model of the two-phase hybrid stepper motor can be obtained by the following set of differential equations $[9,10]$ :

$$
\begin{gathered}
L \frac{d i_{a}}{d t}=-R i_{a}+K_{m} \omega \sin \left(N_{r} \theta\right)+v_{a} \\
L \frac{d i_{b}}{d t}=-R i_{b}+K_{m} \omega \sin \left(N_{r} \theta\right)+v_{b} \\
J \frac{d \omega}{d t}=-i_{a} K_{m} \sin \left(N_{r} \theta\right)+i_{b} K_{m} \cos \left(N_{r} \theta\right)-\tau_{L} \\
\frac{d \theta}{d t}=\omega
\end{gathered}
$$

where, $i_{a}, i_{b}$ and $v_{a}, v_{b}$ are the currents and voltages in phases A and B respectively, $K_{m}$ is the motor torque constant, $\omega$ is the rotor angular speed, $N_{r}$ is the number of rotor teeth, $\theta$ is the rotor position, $L$ and $R$ are the self-inductance and resistance of each phase winding and $\tau_{L}$ is the load torque.

Figure 3 shows the waveform of one phase in each mode.

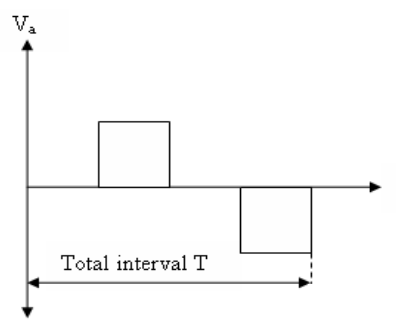

a) Full-Step

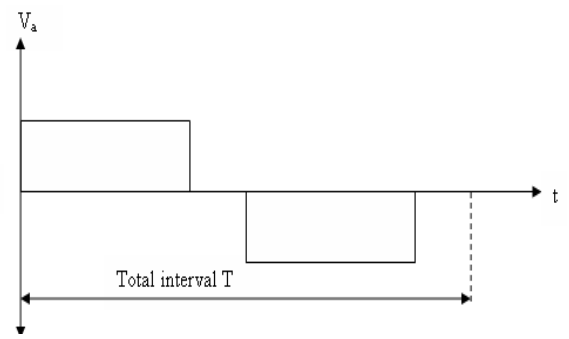

b) Half-Step

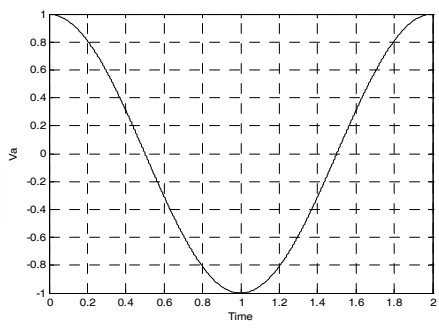

c) Micro-Step

Figure 3 Supply voltage waveform during one complete cycle for one phase 
To compare between different modes, the waveform is analyzed using Fourier series as:

$$
\begin{gathered}
v_{a}(t)=\frac{a_{0}}{2}+\sum_{n=1}^{\infty}\left(a_{n} \cos \frac{2 n \pi t}{T}+b_{n} \sin \frac{2 n \pi t}{T}\right) \\
a_{o}=\frac{2}{T} \int_{0}^{T} v_{a}(t) \cdot d t \\
a_{n}=\frac{2}{T} \int_{0}^{T} v_{a}(t) \cos \left(\frac{2 n \pi t}{T}\right) d t \\
b_{n}=\frac{2}{T} \int_{0}^{T} v_{a}(t) \sin \left(\frac{2 n \pi t}{T}\right) d t
\end{gathered}
$$

Assuming that the fundamental components of the voltage and the current waveforms are:

$$
\begin{aligned}
& v_{a 1}(t)=V_{m} \sin \left(N_{r} \theta+\lambda\right) \\
& i_{a 1}(t)=I_{m} \sin \left(N_{r} \theta+\beta\right)
\end{aligned}
$$

where $V_{m}, \lambda, \beta$, and $I_{m}$ are constants obtained from the Fourier analysis.

Since the electromagnetic torque of the motor resulting from the torques of phase A and B which are given by:

Then,

$$
\begin{gathered}
T_{A}=-K_{m} i_{a} \sin \left(N_{r} \theta\right) \\
T_{B}=K_{m} i_{b} \cos \left(N_{r} \theta\right)
\end{gathered}
$$

And the average torque will be:

$$
\begin{aligned}
& T_{A}=\frac{K_{m} I_{m}}{2}\left[\cos \left(2 N_{r} \theta+\beta\right)-\cos (\beta)\right] \\
& T_{B}=\frac{-K_{m} I_{m}}{2}\left[\cos \left(2 N_{r} \theta+\beta\right)+\cos (\beta)\right]
\end{aligned}
$$

The total torque produced by the stepper motor is:

$$
T_{a v}=-K_{m} I_{m} \cos (\beta)
$$

Since the voltage equation in the phase A is given by: 


$$
v_{a}(t)=i_{a} R+L \frac{d i_{a}}{d t}+e_{a}
$$

where, $e_{a}$ is the induced voltage in the phase winding A and is given by $e_{a}=-K_{m} \omega \sin \left(N_{r} \theta\right)$.

From equations (9) and (10) into equation(18):

$$
V_{m} \sin \left(N_{r} \theta+\lambda\right)=R I_{m} \sin \left(N_{r} \theta+\beta\right)+L I_{m} N_{r} \omega \cos \left(N_{r} \theta+\beta\right)-K_{m} \omega \sin \left(N_{r} \theta\right)
$$

Then, at $N_{r} \theta=\frac{\pi}{2}$

$$
V_{m} \cos (\lambda)=R I_{m} \cos (\beta)-L I_{m} N_{r} \omega \sin (\beta)-K_{m} \omega
$$

And, at $N_{r} \theta=0$

$$
V_{m} \sin (\lambda)=R I_{m} \sin (\beta)+L I_{m} N_{r} \omega \cos (\beta)
$$

Then,

$$
\begin{gathered}
I_{m} \sin (\beta)=\frac{V_{m} \sin (\lambda)-L I_{m} N_{r} \omega \cos (\beta)}{R} \\
I_{m} \cos (\beta)=\frac{V_{m} R \cos (\lambda)+\left(R K_{m}+L N_{r} V_{m} \omega \sin (\lambda)\right)}{\left(R^{2}+L^{2} N_{r}^{2} \omega^{2}\right)}
\end{gathered}
$$

From equations (17) and(23), the motor average torque is:

$$
T_{a v}=-K_{m} \frac{V_{m} R \cos (\lambda)+\left(R K_{m}+L N_{r} V_{m} \omega \sin (\lambda)\right)}{\left(R^{2}+L^{2} N_{r}^{2} \omega^{2}\right)}
$$

This absolute value of torque can be written as:

where:

$$
T_{a v}=\frac{C_{1}+C_{2} \omega}{\left(C_{3}+\omega^{2}\right)}
$$

$$
\begin{gathered}
C_{1}=\frac{K_{m} V_{m} R \cos (\lambda)}{L^{2} N_{r}^{2}} \\
C_{2}=\frac{R K_{m}^{2}}{L^{2} N_{r}^{2}}+\frac{K_{m} V_{m} \sin (\lambda)}{L N_{r}} \\
C_{3}=\frac{R^{2}}{L^{2} N_{r}^{2}}
\end{gathered}
$$




\section{Microcontroller}

The microcontroller in the proposed system is used to:

a) detect quickly the location of the sun at any time by comparing the voltage drop on each LDR;

b) drive the system to the correct position in shortest way and smallest time;

c) control the rotation of each motor; and

d) verify at each sample time that the system is at correct position.

\section{Control Strategy}

The control strategy basically consists of one mode instead of two modes. In this mode, the microcontroller can accurately execute its tasks and then the solar panel can capture the maximum power from the sun for the longest possible time. The control strategy is based on the design of the sun sensor. The sensor is designed as shown in Figure 4.

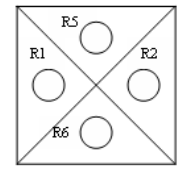

Top view

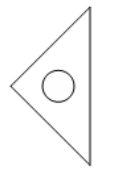

Side view

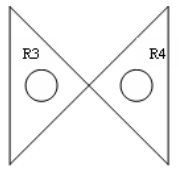

Top view

b) the back side

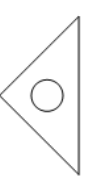

Side view

a) the front side

Design

In this design, six LDRs are used. Four LDRs are located in the front side which shapes a prism. Two LDRs locate in the back side which shapes two triangular. The flowchart shown in Figure 5 has been executed in the microcontroller.

\section{Simulation Results}

To investigate the motor performance, a hybrid stepper motor with parameters given in Table 1 [11] is used as an example to discuss the torque speed characteristics of the motor in full, half and micro-step mode.

Table 1 Hybrid Stepper motor parameters.

\begin{tabular}{|l|l|}
\hline Motor phase resistance & $R=10 \Omega$ \\
\hline Motor phase inductance & $L=0.001 \mathrm{H}$ \\
\hline Machine torque constant & $K_{m}=0.113 \mathrm{Nm} / \mathrm{A}$ \\
\hline Applied DC phase voltage & $V_{s}=v_{a}=v_{b}=5 \mathrm{~V}$ \\
\hline Number of rotor teeth & $N_{r}=50$ \\
\hline
\end{tabular}




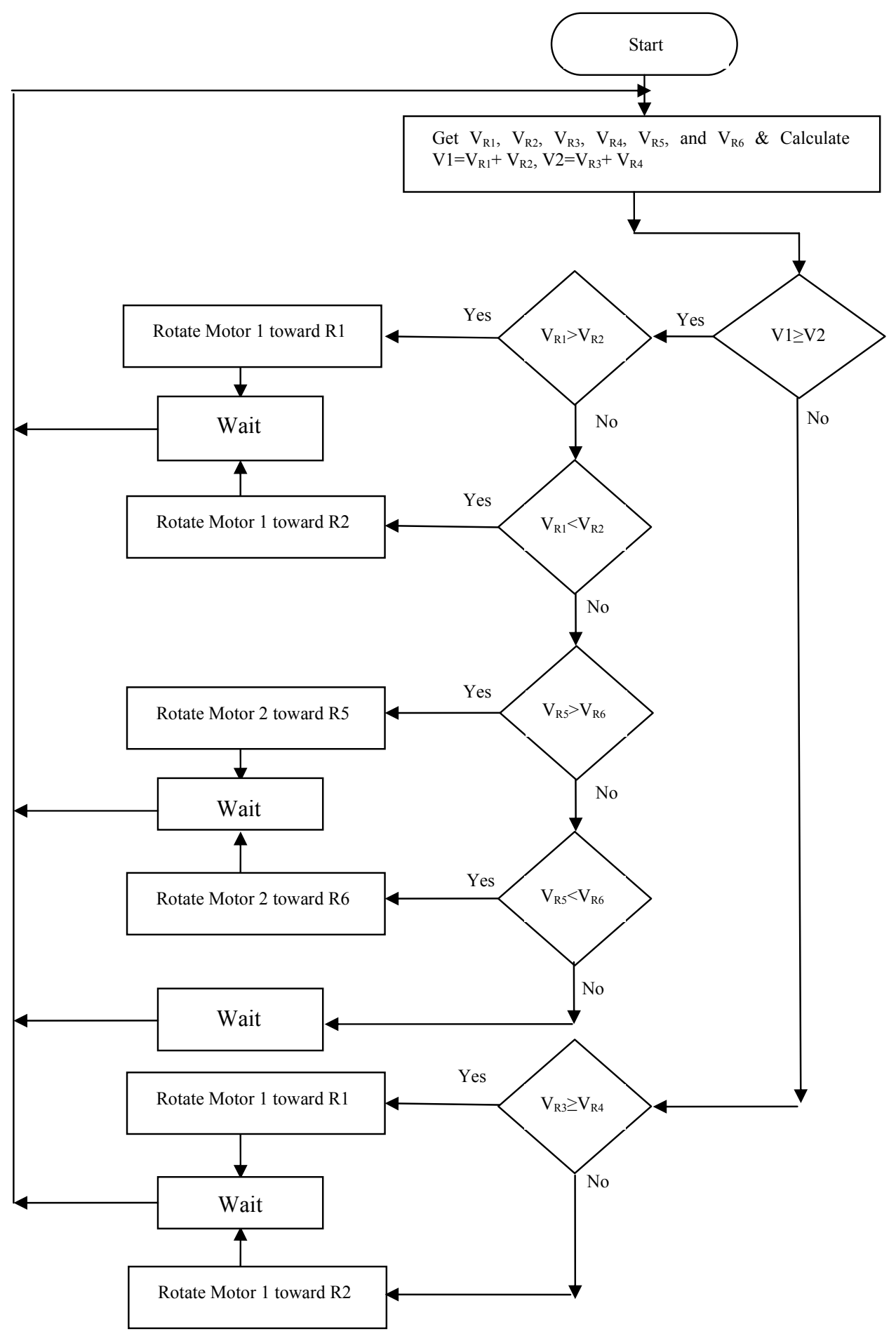

Figure 5 Flowchart of the Control Strategy. 
By analyzing the waveform at different modes, the fundamental voltage component and the average electromagnetic torque are summarized in Table 2.

The analysis relations at different modes are drawn and compared in Figure 6. In Figure 7 the electromagnetic torque obtained in each mode is shown.

Table 2 Analysis of Hybrid Stepper motor at different modes.

\begin{tabular}{|l|l|l|}
\hline Mode of operation & Fundamental voltage component & Average torque \\
\hline Full-step mode & $v_{a 1}(t)=\frac{2 \sqrt{2}}{\pi} V_{s} \sin \left(N_{r} \theta-\frac{\pi}{4}\right)$ & $T_{a v}=\frac{1438.76+43.8822 \omega}{\left(40000+\omega^{2}\right)}$ \\
\hline Half-step mode & $v_{a 1}(t)=1.1763 V_{s} \sin \left(N_{r} \theta+\frac{\pi}{8}\right)$ & $T_{a v}=\frac{2456.05+56.16285 \omega}{\left(40000+\omega^{2}\right)}$ \\
\hline Micro-step mode & $v_{a 1}(t)=V_{s} \sin \left(N_{r} \theta\right)$ & $T_{a v}=\frac{2260+51.076 \omega}{\left(40000+\omega^{2}\right)}$ \\
\hline Proposed mode & $v_{a}(t)=\frac{4}{\pi} V_{s} \sin \left(N_{r} \theta+\frac{\pi}{2}\right)$ & $T_{a v}=\frac{2877.522+51.076 \omega}{\left(40000+\omega^{2}\right)}$ \\
\hline
\end{tabular}

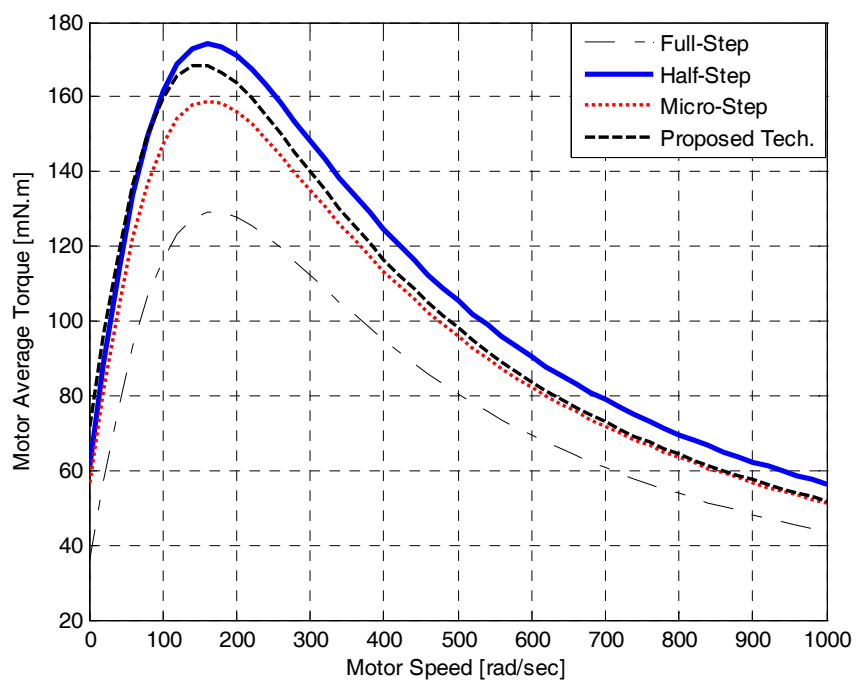

Figure 6 Torque-speed characteristics of Hybrid step motor at different modes. 


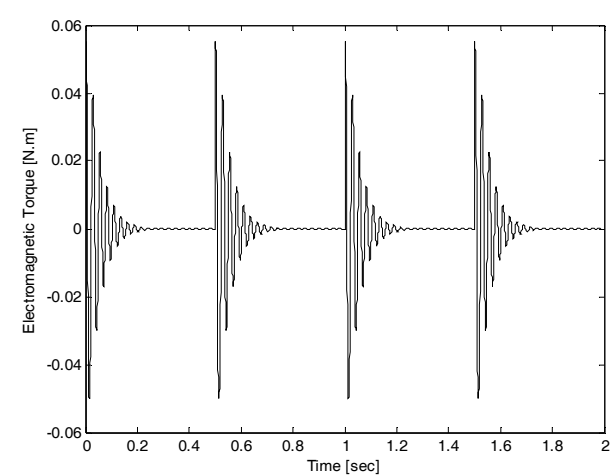

a) Full-Step

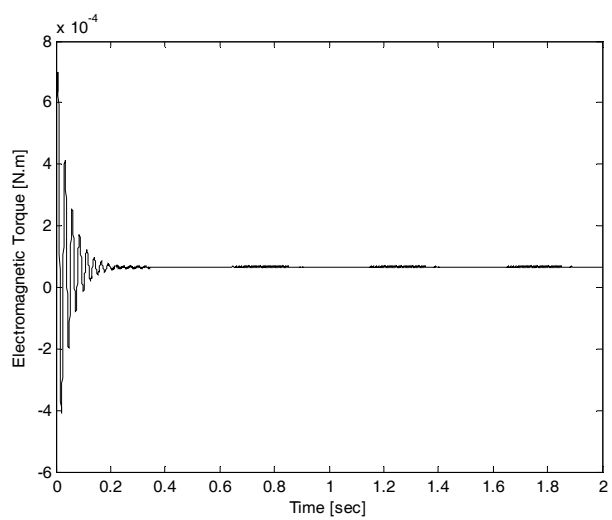

c) Micro-Step

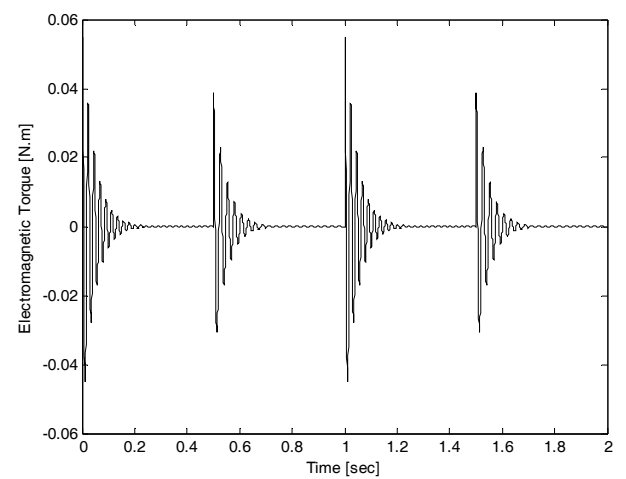

b) Half-Step

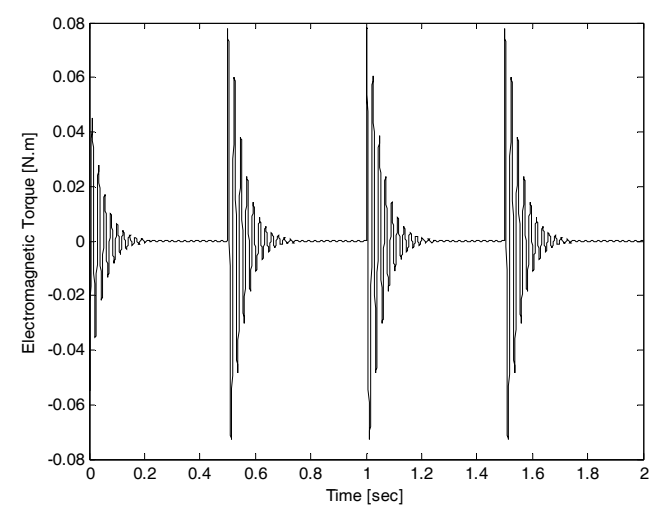

d) Proposed Technique

Figure 7 The Electromagnetic Torque at each step in different modes

From Figure 6, it is clear that the maximum average torque is obtained from the classical halfstep mode. However, Figure 7 shows that the torque available in succeeding steps in case of half-step mode differs and hence will cause different disturbance levels.

Figure 6 shows also that the average torque obtained from the proposed firing technique is higher than that obtained from classical full-step and micro-step modes.

Figure 7 shows that, the torque obtained in each step is symmetrical, so the problem of instability that associated with classical half-step mode is eliminated.

From the flowchart shown in Figure 5, the two hybrid stepper motors cannot rotate at the same time. At the beginning, the microcontroller detects the correct position of the sun. Then, it will rotate the first hybrid stepper motor until the PV module enters the plane that facing the sun. At the end, the second hybrid stepper motor will rotate until PV module capturing the sun. To simulate this action, it is assumed that the sun is at location $18^{\circ}$ and $9^{\circ}$ right and up to the correct position respectively. Figure 8 shows the action of the system where the motor 1 moves 10 steps then motor 2 moves 5 steps. 

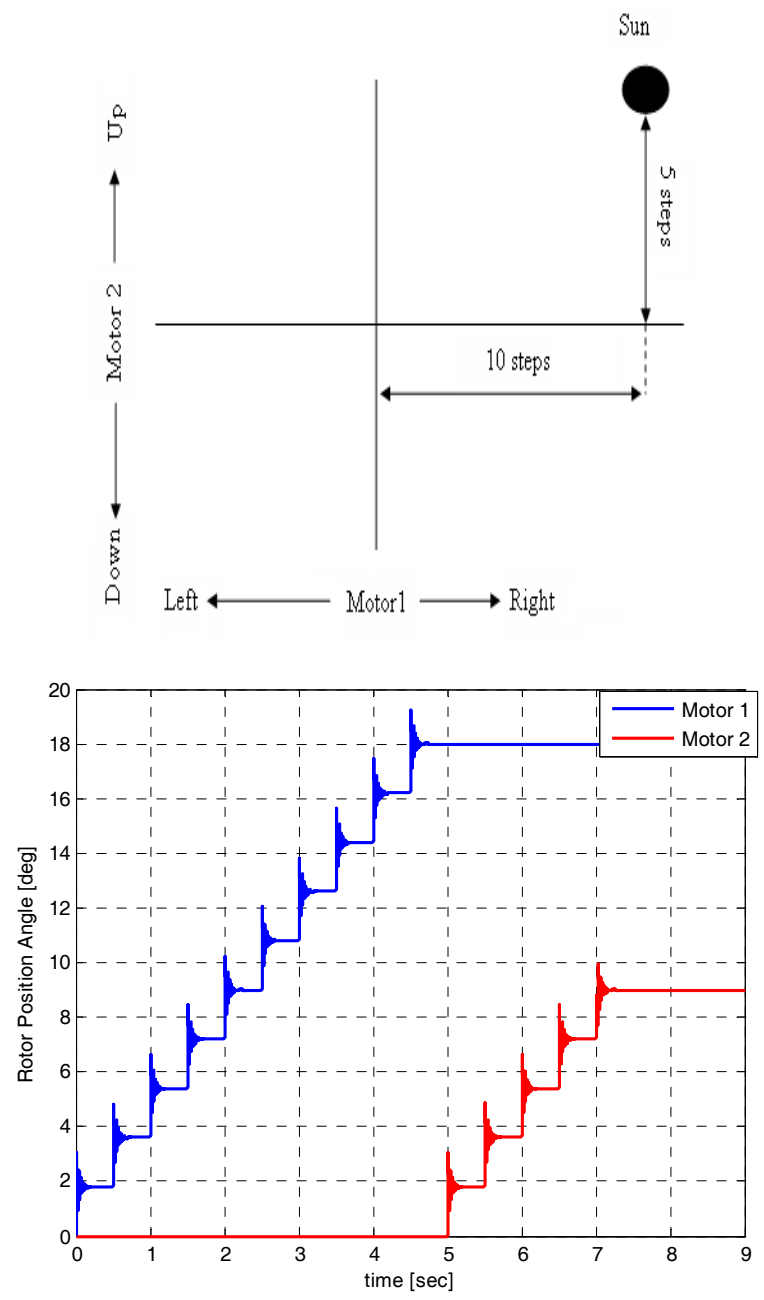

Figure 8 simulation of sun tracker motion.

\section{Conclusion}

The proposed low cost microcontroller based automatic sun tracker that automatically tracks the sun for capturing the maximum solar energy is designed. The controller algorithm operates in one mode and controls two hybrid stepper motors. The hybrid stepper motors are used to replace the classical DC motors. They are analyzed for different operation modes. A full-step mode based on two-phase excitation is proposed to drive the system and provide its highest available torque without any instability problem.

The simulation results presented show the benefits of the proposed firing strategy of hybrid stepper motor with respect to the classical one. They also show the ability of the control strategy to follow the sun without rotation limit. 


\section{References}

[1] Eduardo Román, Ricardo Alonso, Pedro Ibañez, Sabino Elorduizapatarietxe, and Damián Goitia "Intelligent PV Module for Grid-Connected PV Systems" IEEE Transactions On Industrial Electronics, Vol. 53, No. 4, August 2006.

[2] F. Huang, D.Tien, James Or "Microcontroller Based Automatic Sun Tracker Combined with a New Solar Energy Conversion Unit", Proceedings of International Conference on Power Electronic Drives and Energy Systems for Industrial Growth, Vol. 1, 1-3 Dec. 1998, pp: $488-492$.

[3] F.R. Rubio, M.G. Ortega, F. Gordillo, M. Lo'pez-Marti'nez "Application of new control strategy for sun tracking" ELSEVEIER, Energy Conversion and Management, issue 48, 2007, pp: 2174-2184.

[4] P. Roth, A. Georgiev, H. Boudinov "Cheap Two Axis sun following device" Energy Conversion and Management, issue 46, 2005, pp: 1179-1192.

[5] C. Aracil, J. M. Quero, L. Castañer†, R. Osuna, and L. G. Franquelo "Tracking system for solar power plants", 32nd Annual Conference on Industrial Electronics, IECON 2006 - Volume , Issue , 6-10 Nov. 2006, pp:3024 - 3029.

[6] A Robust Position Estimation Scheme Using Sun Sensor, IEEE Transactions on Instrumentation and Measurement, Volume 47, Issue 2, Apr 1998, pp:595 - 603.

[7] M.Zribi, J. Chiasson "Position control of PM stepper motor by exact linearization", IEEE Transactions on Automatic Control, Vol. 36, Issue 5, May 1991, pp:620 - 625

[8] K. R. Rajagopal, M. Krishnaswamy, Bhim Singh, and B. P. Singh "An Improved HighResolution Hybrid Stepper Motor for Solar-Array Drive of Indian Remote-Sensing Satellite" IEEE Transactions on Industry Applications, vol. 33, no. 4, July/August 1997

[9] Ja.Avarez-Gallegos, E. Alvarez-Sanchez and R.chastro-Linares "Experimental Setup for the Sensorless Rotor Position Control of a Permanent Magnet Stepper Motor", Power Electronics Congress, 2004. CIEP 2004. 9th IEEE International Publication Date: 17-22 Oct. 2004, pp: 123- 127.

[10] Sukumar Kamalasadan "A New Intelligent Controller for the Precision Tracking of Permanent Magnet Stepper Motor", IEEE Power Engineering Society General Meeting, Vol. I, 24-28 June 2007, pp:1 - 7.

[11] Hamdy Abd El-Halim Mohammad, "ANALYSIS AND POSITION CONTROL OF STEPPER MOTOR USING ARTIFICIAL INTELLEGENCE”, PhD thesis, Al-Azhar University, 2006. 